\title{
Fault-Current Injection Strategies of Inverter-Based Generation for Fast Voltage Recovery
}

\author{
Stefan Stanković, Student Member, IEEE, Thierry Van Cutsem, Fellow, IEEE and Lennart Söder, Senior Member, \\ IEEE
}

\begin{abstract}
As the inverter-based generation replaces the conventional synchronous generators, it may also need to fill in the missing ancillary service support. One of these ancillary services is dynamic reactive power provision and voltage control. This paper analyzes optimal strategy of reactive and active fault-current support of the inverter-based generation leading to fast voltage recovery of the system. For the purpose of the analysis, new ramping active current controller able to emulate different behavior of active current injection is proposed. By optimizing its parameters for different case studies of the system, the conclusions about optimal behavior of the inverter based generation with respect to system parameters and operating conditions are drawn. It is observed that the optimal combination of active and reactive fault-current is the most sensitive to the dynamic load component penetration levels in the system. With the increasing penetration levels, the significance of active faultcurrent injection increases. The results show that with higher penetration levels of dynamic load component in the heavy load areas, the ramping down of the inverter-based generation active fault-current results in slower voltage recovery of the system. Following this conclusion, a recommendation on update of current European grid codes is proposed.
\end{abstract}

Index Terms-voltage recovery, inverter-based generation, dynamic system optimization, voltage stability

\section{INTRODUCTION}

The transition from conventional to renewable energy sources leads to increased share of Inverter-Based Generation (IBG) in power systems. As synchronous generators are being replaced with IBGs, the power systems experience decreased provision of ancillary services such as reactive power support. One of the reasons for this is the worse ability of IBGs to be overloaded for the short period of time supplying higher currents to the systems compared to synchronous generators. This ability is especially important during and after the occurrence of a fault in the system. By supplying high enough values of reactive currents, the generators can support the voltages from dipping too much and consequently provide faster voltage recovery with higher short-circuit capacity for the system. On the other side, IBGs have a very limited ability to do so.

A common way to increase the reactive current injection during the fault and immediately after the fault is to decrease or even cut-off active current injections of IBGs. This sudden increase of reactive current may also pose a risk of overvoltages in the system if the reactive current is not decreased fast

Stefan Stanković and Lennart Söder are with the Division of Electric Power and Energy Systems, School of Electrical Engineering and Computer Science, KTH Royal Institute of Technology, Stockholm, Sweden (e-mail: stanko@kth.se; 1sod@kth.se)

Thierry Van Cutsem is with the Fund for Scientific Research (FNRS) at the University of Liège, Belgium (e-mail: t.vancutsem@uliege.be) enough after the fault clearing. The solution of prioritizing reactive current in front of active current during the fault has been implemented in a number of grid codes in Europe [1]-[4]. In some others, like the one from Irish Transmission System Operator (TSO) EirGrid [5], the requirements are set on prioritizing the active current during the fault. Danish grid code allows TSO to decide on case-dependent appropriate settings of IBGs fault-currents injection [6]. These different requirements in the grid codes of different countries pose an interesting research question on why and how should the faultcurrents of the IBGs be controlled. This paper addresses this question from the perspective of their influence on the speed of voltage recovery of the system.

IBGs current control and its influence on the fault-induced response of the system has been analyzed in [7]-[12]. Some of the papers analyze this influence considering IBGs as a part of microgrids [7], [8] while the others, including also this paper, consider IBGs as stand-alone units [9]-[12]. The first ones focus on sharing of active and reactive current contribution of individual IBGs in a microgrid during the fault and their capability to follow the reference power exchange with the overlying grid. The second ones focus more on how a certain IBG current control strategy affects a fault-induced dynamic response of the overlying system. The most commonly considered IBG current control is voltage droop controller on reactive current [9] or reactive power [10], [12] injection of IBG. This controller assumes reactive current priority and is proposed by most of the European grid codes [1]-[4]. During the faults which lead to very low system voltages, this controller may lead to full cut-off of IBG active current. After the fault is cleared, the active current is recovered to the pre-fault value. The speed of the active current recovery and its influence on the dynamic response of the system has been analyzed in [10]. Another, more complex IBG current controller design, proposed by [11], uses the information on effective $R / X$ ratio at the connection point of the IBG to calcuate references of active and reactive current injection. All these controllers have in common that they to some extent require ramping up or down of active current injection during the fault to accommodate the need of reactive current controller. However, the influence of IBG active current ramping during the fault on the later speed of system voltage recovery has not been clearly investigated. This paper analyzes optimal strategies of controlling IBG active fault-currents, with regards to different system setups, to achieve fast fault-induced voltage recovery of the system.

For the purpose of the analysis, a new IBG current controller structure is proposed with priority to the active current. It 
consists of an active current ramping controller and a reactive current controller similar to the one described in the grid codes [1]-[4] and previously considered in [9]. By adjusting the ramp coefficient of the active current controller, different scenarios of active fault-current control can be assessed e.g. constant current control and full cut-off of the active current during the fault.

The primary goal of the analysis reported in this paper is to find the optimal ramp coefficients with respect to some of the most relevant parameters of the system i.e. investigate what is the optimal way to control active fault-current of the IBG for different values of system parameters. The system parameters considered in the analysis are different $\mathrm{R} / \mathrm{X}$ ratios of the lines in the area where IBGs are installed and different ratios between dynamic and static load components. The optimal ramp coefficients do not show a large sensitivity to different $\mathrm{R} / \mathrm{X}$ ratios but do show significant sensitivity to the different ratios of the load components. To further investigate this sensitivity of the optimal controller settings to the uncertainty of the load parameters, Monte Carlo analysis is used. The analysis shows that a certain accuracy of load modeling is needed for optimal tuning of the proposed controller yielding the optimal or near optimal system voltage recovery. This result is in line with the recent research done on load and Active Distribution Network (ADN) modeling for the purpose of system dynamics studies [13], [14].

As a side contribution and for the purpose of obtaining optimal parameter settings of the proposed controller, this paper also proposes using of desirable parameter space to represent the optimal solution when the search space is noisy and the model parameters are uncertain. Desirable parameter space represents a convex polytope which wraps the cluster of points in the search space whose relative value of the objective function is not much worse than the one of the optimum point. Since for the optimization part of the analysis in this paper the genetic algorithm combined with clustering techniques [15] is used, the polytope wraps the cluster of chromosomes containing the globally best chromosome.

Based on the results of the analyzes of this paper, a possible update of certain grid codes is proposed. The paper is organized into following sections:

1) Introduction - gives a brief description of the problem, research gap and main contributions of the paper;

2) Problem setup - sets up the problem of finding optimal IBG active current control strategies for fast voltage recovery and introduces methods used to solve it;

3) Case study - presents results of case studies with accompanying robustness analysis of proposed control designs;

4) Discussion - discusses results of the paper, limitations of the studies and future work;

5) Conclusion - concludes the paper.

\section{PROBLEM SETUP}

The problem of finding the values of certain parameters of the system, e.g. controller settings, that would lead to the fastest voltage recovery after a fault can be formulated as a dynamical system optimization problem. The objective is to optimize the trajectory of certain state variable over the observed period of time i.e. the fastest recovery of the pilot bus (representative bus of the voltage control area) voltage to its pre-fault value. In general, this problem can be written as:

$$
\begin{array}{ll}
\min & \varphi_{v}(x) \\
\text { s.t. } & \dot{f}\left(t, x, x_{d}, p, u_{p}\right)=0, \\
& g\left(t, x, x_{d}, p, u_{p}\right)=0, \\
& h\left(t, x, x_{d}, p, u_{p}\right) \geq 0,
\end{array}
$$

where the dynamics of system are described by the set of differential equations $\dot{f}$, algebraic equations $g$ and nonequalities $h$. All of these relations are given as functions of time $t$, continuous $x$ and discrete system states $x_{d}$, constant set of parameters of the system $p$ and the set of system parameters used as decision variables in the optimization problem $u_{p}$. The objective function $\varphi_{v}$ should be chosen such that its minimization reflects faster voltage recovery. In this paper we use the formulation proposed by [16]. This formulation represents the voltage response curve at the pilot buses as the Probabilistic Density Functions (PDF) describing how much time the voltages were at the certain level during the observed response. In order to get the value of $\varphi_{v}$, these PDFs are compared with the Dirac distribution, representing the ideal voltage recovery, using the Kullback-Leibler (KL) divergence criteria.

To improve the voltage recovery of the system, IBGs ability to control the injection of active and reactive current during and in the seconds after the fault is cleared can be used. For the purpose of analyzing of different IBG active faultcurrent control strategies and their influence on the speed of the voltage recovery of the system, this paper proposes a new IBG's current controller structure and provides a procedure for optimal tuning of its parameters given as $u_{p}$ in (1). Before describing the dynamical model of the proposed controller in subsection II-B and the optimal tuning procedure in subsection II-C, the load models are presented.

\section{A. Load modeling}

When it comes to the analysis of the voltage dynamics of power systems, an important part of the system that requires careful modeling are the loads [17], [18]. The load model used in the analyses in this paper consists of three components:

1) static exponential load model;

2) dynamical load model depicting small induction motors and

3) dynamical load model depicting large induction motors.

Active $P_{s l}$ and reactive power $Q_{s l}$ of the static component of the load model are given by the well-known exponential model:

$$
\begin{aligned}
P_{s l} & =P_{s l 0}\left(\frac{U}{U_{n}}\right)^{\alpha} \\
Q_{s l} & =Q_{s l 0}\left(\frac{U}{U_{n}}\right)^{\beta}
\end{aligned}
$$

The dynamical model of the induction motor is a two-axis model with only rotor circuit dynamics included, commonly 
TABLE I

PARAMETERS OF THE INDUCTION MACHINE MODELS

\begin{tabular}{|c|c|c|c|c|c|c|c|c|c|}
\hline \multirow{2}{*}{$\begin{array}{c}\text { type of } \\
\text { machine }\end{array}$} & \multicolumn{10}{|c|}{ parameters } \\
\cline { 2 - 10 } & Rs & Lls & Lm & RR & Llr & H & A & B & LF \\
\hline small machine & 0.031 & 0.100 & 3.2 & 0.018 & 0.180 & 0.7 & 0 & 0 & 0.6 \\
\hline large machine & 0.013 & 0.067 & 3.8 & 0.009 & 0.170 & 1.5 & 0 & 0 & 0.8 \\
\hline
\end{tabular}

used for power system stability studies [18]. This model is defined by the following parameters:

- Rs [pu] - stator resistance;

- Lls [pu] - stator leakage inductance;

- Lm [pu] - magnetizing inductance;

- $\operatorname{Rr}[\mathrm{pu}]$ - rotor resistance;

- Llr [pu] - rotor leakage inductance;

- H [s] - inertia constant;

- A, B - coefficients defining the mechanical torque characteristics $T_{m}=T_{m 0}\left(A \Omega_{m}^{2}+B \Omega_{m}+1-A-B\right)$ where $\Omega_{m}$ is mechanical rotational speed of the shaft;

- LF - load factor defined as $L F=P_{m} / S_{n}$ where $P_{m}$ is the active power drawn by the motor in steady state and $S_{n}$ its nominal apparent power.

The chosen values of these parameters defining small and large induction machine are given in Table I taken from [19].

The total active and reactive powers of the load are decomposed into:

$$
\begin{aligned}
P_{l} & =a P_{s l}+b P_{s m}+(1-a-b) P_{l m} \\
Q_{l} & =a Q_{s l}+b Q_{s m}+(1-a-b) Q_{l m}
\end{aligned}
$$

where $P_{s m}$ and $Q_{s m}$ are the active and reactive powers of the small induction motor, and $P_{l m}$ and $Q_{l m}$ the corresponding powers of the large induction motor. Coefficients $a$ and $b$ define the ratio of static, small and large machine load, respectively. The amount of dynamic load component is given in percentage of the total load as $\gamma=(1-a) 100 \%$.

\section{B. Ip/Iq controller}

The dynamical model of IBG is taken from [20] and modified to include the proposed Ip/Iq controller for the fast voltage recovery. The main features of the original model are:

1) generic PLL model;

2) voltage measurement block represented as a first order transfer function with time constant $T_{m}=0.01 \mathrm{~s}$;

3) converter dynamics represented as a first order transfer function with the time constant $T_{g}=0.02 \mathrm{~s}$;

4) limiters on converter currents with defined active or reactive current priority when the maximum converter current is reached.

The changes to the original model are the following:

1) new supplementary action on active current injection $I_{p}$;

2) new supplementary action on reactive current injection $I_{q}$;

3) no primary frequency support from the IBG.

The $I_{p}$ supplementary control was implemented as a ramping controller with the output shown in Fig. 1. The ramp slope is defined by:

$$
k_{I p}=\frac{\Delta I_{p}}{t_{o f f}-\Delta t_{1}-t_{o n}}
$$

The shape of the $I_{p}$ supplementary control output is defined and can be adjusted by changing the parameters $\Delta t_{1}, \Delta t_{2}$ and $k_{I p} . \Delta t_{1}$ is the time delay after local identification of the fault at time instant $t_{\text {on }}$ until the start of the active current ramping. $\Delta t_{2}$ is the time after the fault clearing detection at time instant $t_{\text {off }}$ until the supplementary active current reference is reset to zero.

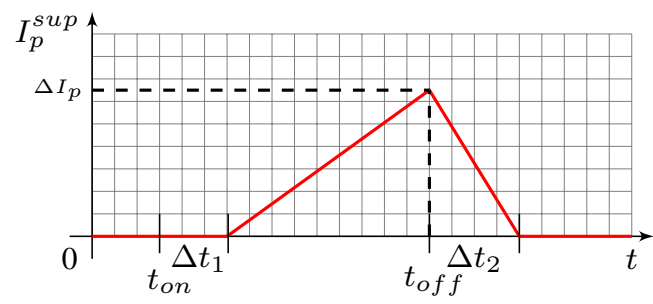

Fig. 1. Supplementary $I_{p}$ ramping controller

The $I_{q}$ supplementary control is given as a proportionaldifferential controller shown in Fig. 2 whose input is the filtered voltage measurement $V_{m}$. The voltage setpoint $V_{\text {ref }}$ is chosen to be pre-fault voltage at the IBG bus. The proportional gain $k_{q}$ represents the droop controller commonly recommended in the grid codes for supplementary reactive current injection [9]. The grid codes also usually propose that the supplementary control is activated when the voltage falls below certain value. Our proposed controller acts over whole voltage range. Another difference is that the value of the gain in this case is much higher than usually recommended to achieve faster response during the transients. The differential action is added here to damp possible oscillations that may occur with higher proportional gain values $k_{q}$. If no such oscillations appear in the system, the derivative gain $k_{q}^{d}$ could be set to zero. To avoid negative effects of derivative action, a filter with time constant $T_{q}^{f}$ is used and the output is limited. $T_{q}^{f}$ should be appropriately chosen in order not to filter out the dynamics of interest.

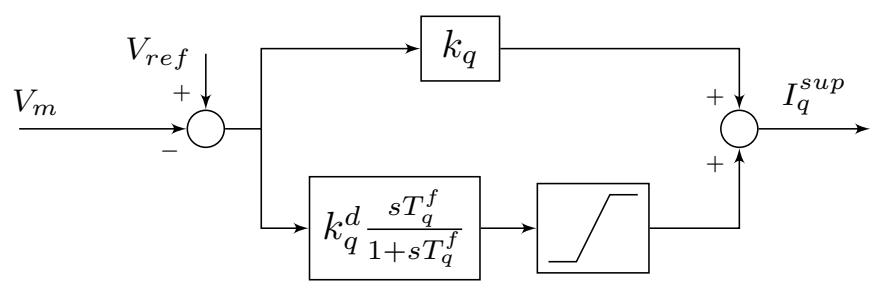

Fig. 2. Supplementary action on IBG's reactive current injection $I_{q}$

Note that the current injection priority of the controller is set to the active component $I_{p}$. This means that the upper and lower limits on reactive current injection are dictated by the nominal current $I_{n}$ of the controller and the current value of active current $I_{p}$.

The idea behind the proposed controller is that it acts only in emergency situations. The proposed controller gets activated when the sudden drop of voltage is detected locally indicating contingency in the system that may lead to prolonged depression of local voltages. After the full recovery of the voltage indicating the end of emergency, the proposed controller is 
disabled and the control is given to the IBG current controllers designed to work in the normal operating conditions.

\section{Optimization of controller parameters}

Having the structure of the proposed controller, it is of interest to find how to optimally set its parameters to achieve the fastest possible voltage recovery of the system given a set of various pre-fault operating conditions. As mentioned, such a task can be considered as a dynamical system optimization problem given by (1) where the parameters of the proposed controller to be tuned are decision variables $u_{p}$. This kind of optimization problems can be solved using direct methods based on discretization of differential equations in (1) [21] or indirect methods which use calculus of variations. The second ones have been reported to poorly handle the inequality constraints [22]. Also, their application on complex and high dimensional problems might be very difficult [23]. Therefore, in this paper a direct method based on partial discretization is chosen.

Partial discretization discretizises only the control variables in (1). The values of control variables for each time step are considered as decision variables in the optimization problem. Then, at each iteration of the optimization routine, the values of the system states over the observed time interval are found by a DAE solver for the values of decision variables in the current iteration. In the analysis in this paper, the control variables converted to decision variables are the controller parameters $u_{p}$, and they are considered constant over the simulated time interval. The DAEs of the system dynamical model have been solved using the RAMSES phasor-mode simulation software [24].

The previously described optimization problem is in general a nonconvex problem, with discontinuities and numerical noise in the search space. Numerical noise mostly comes from the objective function in (1) [16] but also from the finite precision of the DAE solver. Therefore, gradient-based optimization methods would be prone to failing in finding optimal values of $u_{p}$. As an alternative, the optimization method based on Genetic Algorithms and clustering techniques described in [15] has been used in this study.

Having the search space with possible flat areas smeared with numerical noise brings a question on how reliable is the information about the single optimum point as a solution. Further on, such a solution is based on the perfect knowledge of the system parameters and simplified model that only approximates reality. The proposed optimization method, besides the optimal solution point, also gives the cluster of the nearby chromosomes (points in the search space) surrounding the identified optimum. Such a cluster represents a part of the search space in which the value of the objective function does not change a lot compared to the rest of the search space. This cluster is further on referred as Desirable Parameter Space (DPS) and it is given as an $N$-dimensional convex polytope, where $N$ is number of elements in a set $u_{p}$. The polytope wraps all the chromosomes belonging to the same cluster as the identified optimum.

The identification of the area surrounding the possible optimum, given as a DPS, might be more important than the
TABLE II

DATA OF THE DIFFERENT FEEDER TYPES HOSTING IBGS

\begin{tabular}{|c|c|c|c|c|c|c|}
\hline \multicolumn{2}{|c|}{ feeders } & \multicolumn{2}{|c|}{ load [MW/MVAr] } & \multicolumn{3}{|c|}{ generation [MW/MVAr/MVA] } \\
\hline name & bus no. & $P_{l}$ & $Q_{l}$ & $P_{g}$ & $Q_{g}$ & $S_{g}$ \\
\hline \multirow{5}{*}{ LL } & 1 & I & I & 25.00 & 0.00 & 30.00 \\
\hline & 2 & 80.00 & 20.00 & l & I & I \\
\hline & 3 & 50.00 & 10.00 & l & l & I \\
\hline & 4 & I & I & 30.00 & 0.00 & 35.00 \\
\hline & 5 & 50.00 & 10.00 & I & I & I \\
\hline \multirow{5}{*}{ SL } & 1 & 30.00 & 10.00 & I & I & 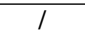 \\
\hline & $2 *$ & I & I & 30.00 & 5.00 & 45.00 \\
\hline & 3 & 40.00 & 15.00 & l & I & I \\
\hline & 4 & 30.00 & 10.00 & I & I & I \\
\hline & 5 & I & I & 20.00 & 5.00 & 25.00 \\
\hline \multirow{5}{*}{$\mathrm{LG}^{*}$} & 1 & I & I & 80.00 & $\begin{array}{c}30 \\
(-15)\end{array}$ & 100.00 \\
\hline & 2 & 40.00 & 10.00 & I & I & I \\
\hline & 3 & I & I & 50.00 & $\begin{array}{c}20 \\
(-10)\end{array}$ & 80.00 \\
\hline & 4 & I & I & 50.00 & $\begin{array}{c}20 \\
(-10)\end{array}$ & 80.00 \\
\hline & 5 & 30.00 & 5.00 & I & I & I \\
\hline \multirow{5}{*}{ SG } & 1 & I & I & 30.00 & 0.00 & 35.00 \\
\hline & 2 & I & I & 30.00 & 0.00 & 35.00 \\
\hline & 3 & 30.00 & 10.00 & I & I & I \\
\hline & 4 & 30.00 & 10.00 & I & I & I \\
\hline & 5 & I & I & 20.00 & 0.00 & 25.00 \\
\hline
\end{tabular}

identification of the optimum as a single point in the search space. The case study in the following section illustrates this in a greater detail.

\section{CASE STUDY}

\section{A. Test system}

As a basis for the case study, the IEEE Nordic test system, set up by the IEEE Task force on test systems for voltage stability and security analysis, has been used in this study. The secure operating point B detailed in [25] has been selected. Modifications of the system are done to the $130-\mathrm{kV}$ grid in the Central area containing buses 1041, 1042, 1043, 1044 and 1045. Generators, loads and shunts at buses 1041, 1042 and 1043 are replaced with radial $130-\mathrm{kV}$ grids hosting distributed IBGs and loads, in such a way that the operating point B is preserved in the rest of the system. Each radial grid is assembled as a combination of four types of feeders consisting of the Point of Common Coupling (PCC) bus and five other buses with the power injections data given in Table II. Schematics of the introduced radial grids are shown in Fig. 3.

The sections in all the feeders are assumed to be 6-km long with two considered physical realizations: overhead lines and cables. In order to preserve operating point B of the system, shunt capacitors are added at buses 1041 and 1043 with the nominal powers given in Table III. In the case of the cable feeders, the reactive power set-points of the generators in LG feeders are set to the values given in parentheses in Table II. Final adjustments to match the operating point B consist of fine tuning the active and reactive powers of the generators at bus 2 in each SL feeder.

The load models used in the case study have been described in subsection II-A. The values of the exponents $\alpha$ and $\beta$ are 


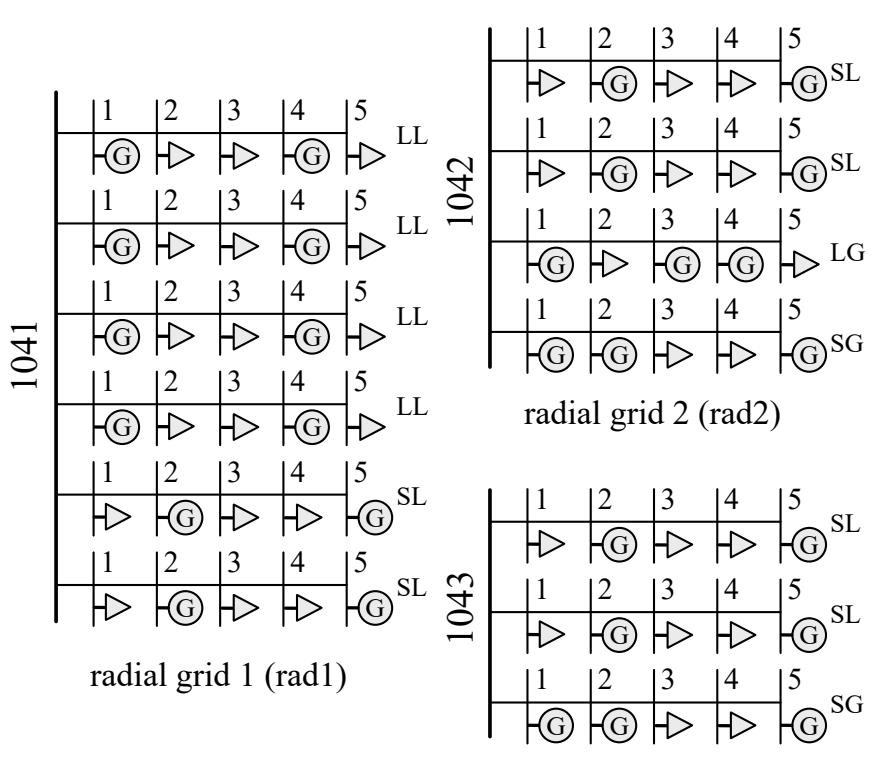

radial grid $3(\operatorname{rad} 3)$

Fig. 3. Additional radial grids connected at $130-\mathrm{kV}$ buses

TABLE III

SHUNT CAPACITOR SIZES FOR DIFFERENT PHYSICAL REALIZATIONS OF THE FEEDERS

\begin{tabular}{|c|c|c|}
\hline shunt [MVAr] & \multicolumn{2}{|c|}{ case study } \\
\cline { 2 - 3 } connected at bus & overhead lines & cables \\
\hline 1041 & 300 & 100 \\
\hline 1043 & 200 & 120 \\
\hline
\end{tabular}

chosen to be 2 for all loads except the ones directly represented at $130-\mathrm{kV}$ whose exponent $\alpha$ is set to 1 . Also, in dynamic simulations, for voltage values lower than $0.5 \mathrm{pu}$ (resp. 0.7 $\mathrm{pu}$ ) the loads connected to $130-\mathrm{kV}$ (resp. to the other) buses become constant impedance loads.

In order to investigate the proposed IBG current controller's performance and tuning sensitivity to different system parameters, a total of six scenarios are created. As already mentioned, two different physical realizations of the radial feeders have been considered: overhead lines $(\mathrm{OH})$ and cables $(\mathrm{Cab})$. Some recent studies showed the importance of load and distribution systems equivalent modeling for proper assessment of power system dynamics [13], [14]. Therefore, for both $\mathrm{OH}$ and Cab realizations, the influence of different ratios between dynamic and static load components has been assessed: $\gamma=10 \%, 20 \%$ and $30 \%$. To refer to specific case study scenario, the following notation is used. For example, a scenario with overhead line feeders and loads having $\gamma=20 \%$ of dynamic component is referred as $\mathrm{OH}-20$ scenario. Each scenario is subjected to two different faults:

F1: three-phase short circuit at bus 4045 that is cleared after $100 \mathrm{~ms}$ by opening the line 4044-4045 and

F2: three-phase short circuit at bus 1044 that is cleared after $100 \mathrm{~ms}$ without opening any lines.

Both faults can induce depressed voltages in $130 \mathrm{kV}$ grid and delayed voltage recovery of the system.

\section{B. Optimization Setup}

Starting from the proposed structure of the controller described in subsection II-B, the goal of the analysis is to find values of ramping parameters $k_{I p}$ that solve the problem of the fastest voltage recovery defined by (1). The other two parameters of the ramp in Fig. 1 are kept constant at values $\Delta t_{1}=0$ and $\Delta t_{2}=0$. This choice is based on the results of the previous studies [9], [10] which show that reacting as fast as possible after the fault and restoring the IBG active current to the pre-fault value yields the best voltage recovery.

To reduce the number of decision variables and make the optimization more efficient, the IBGs located in the assembled radial grids are divided into eight groups where the IBGs from the same group have the same settings of the ramping parameter $k_{I p}$. These groups, given in Table IV, are formed based on similar positions of the IBGs i.e. similar electrical distance from a PCC and the same hosting feeder type. Each group $i$ has been attributed a decision variable $k_{I p}^{i}$.

TABLE IV

IBG GROUPS WITH THEIR CORRESPONDING RAMP PARAMETERS $k_{I p}^{i}$

\begin{tabular}{|c|c|c|c|c|c|}
\hline group no. & total IBGs & total MVA & radial grid & feeder type & buses no. \\
\hline 1 & 4 & 120 & $\operatorname{rad} 1$ & LL & 1 \\
\hline 2 & 4 & 140 & $\operatorname{rad} 1$ & LL & 4 \\
\hline 3 & 2 & 90 & $\operatorname{rad} 1$ & SL & 2 \\
\hline 4 & 2 & 50 & $\operatorname{rad} 1$ & SL & 5 \\
\hline 5 & 1 & 45 & $\operatorname{rad} 3$ & SL & 2 \\
\hline 6 & 1 & 25 & $\operatorname{rad} 3$ & SL & 5 \\
\hline 7 & 1 & 70 & $\operatorname{rad} 3$ & SG & 1,2 \\
\hline 8 & 1 & 25 & $\operatorname{rad} 3$ & SG & 5 \\
\hline
\end{tabular}

The IBG groups with their corresponding parameters $k_{I p}$ lead to an 8-dimensional vector $u_{p}$ in (1). The objective function of (1) is defined by the voltage response at a single pilot bus, namely bus 1041. This bus is chosen as it is the weakest in terms of voltage fluctuations, as shown in [25].

The IBGs connected to the radial grid 2 are not involved in the optimization, and their active current injections are considered to be constant. This is because it can be observed that bus 1042, to which the radial grid 2 is connected, is electrically quite far compared to buses 1041 and 1043 . Another reason is that the grid connected to bus 1042 has enough reactive power capacity to control the voltage at bus 1042, making the optimization of the active current injection not a problem of interest when it comes to the fast voltage recovery at bus 1042 .

For the purpose of optimization, ordinary genetic algorithm [26], [27] is used. The parameters of the algorithm are given in Table V. Chromosomes are represented in a binary format using gray code to avoid creation of hamming walls in the search space. For the selection phase, combination of roulette wheel and elitism techniques are chosen. To avoid premature convergence and enhance algorithm convergence, fitness functions of chromosomes are scaled before the selection phase. For this purpose linear, power and sigma scaling [26], [27] are used. After the selection, multi-point crossover is applied with a number of crossover points equal to the number of decision variables represented as genes in each chromosome. 

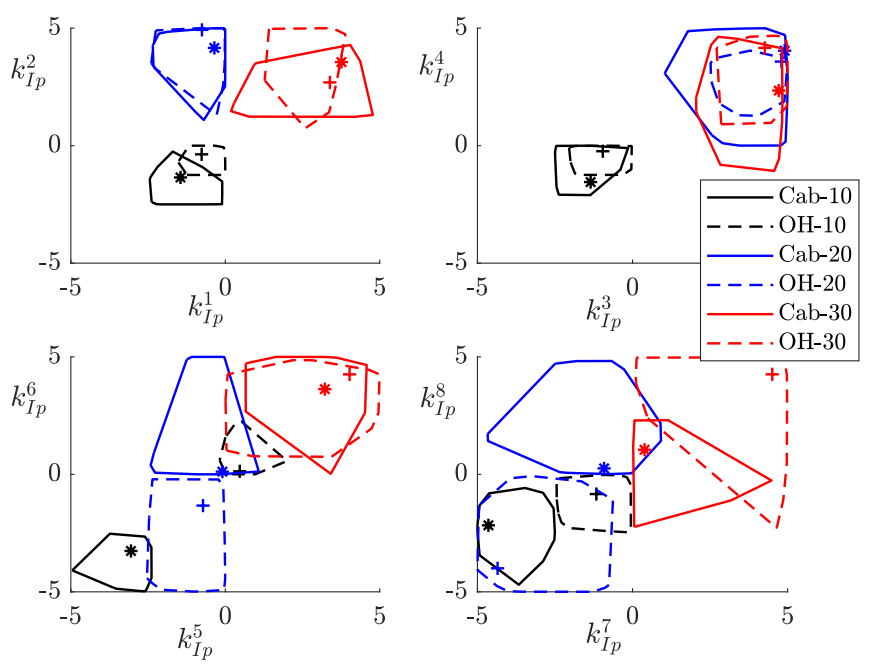

Fig. 4. Optimal values of ramp parameters $k_{I p}^{i}$ with their associated DPS for different analyzed scenarios in case of fault F1 at bus 4045

The location of the crossover points is selected randomly for each pair of parent chromosomes. As a stopping criteria, a maximum number of generations is chosen. To identify the chromosomes clusters and create DPSs, DBscan [28] clustering technique is used.

TABLE V

PARAMETERS OF THE GENETIC ALGORITHM

\begin{tabular}{cc}
\hline Parameter & Value \\
\hline Number of chromosomes & 450 \\
Number of genes & 8 \\
Number of alleles & 12 \\
Generation gap & 0.7 \\
Probability of crossover & 0.8 \\
Probability of mutation & 0.0004 \\
Maximum number of generations & 30 \\
Fitness linear scaling coefficient & 1 \\
Fitness power scaling coefficient & 3 \\
\hline
\end{tabular}

\section{Optimization Results}

By solving the problem (1) for the various scenarios of our case study, using the optimization method outlined in Subsection II-C, the optimal $k_{I p}$ values with their associated DPS are obtained. Their projections onto four two-dimensional parameter spaces are shown in Fig. 4 for the case of fault F1 at bus 4045 and in Fig. 5 for the case of considered fault F2 at bus 1044. The projections of the DPS are given as convex polygones while the optimum points belonging to the same colored DPS are given as stars in the case of cables and pluses in the case of overhead lines. Analyzing the shape and the position of the DPS projections, a number of conclusions regarding sensitivity of optimal $k_{I p}^{i}$ settings to the system parameters and IBG locations can be drawn as follows.

As explained in subsection II-C, the DPS represents a part of the search space in which the objective function does not
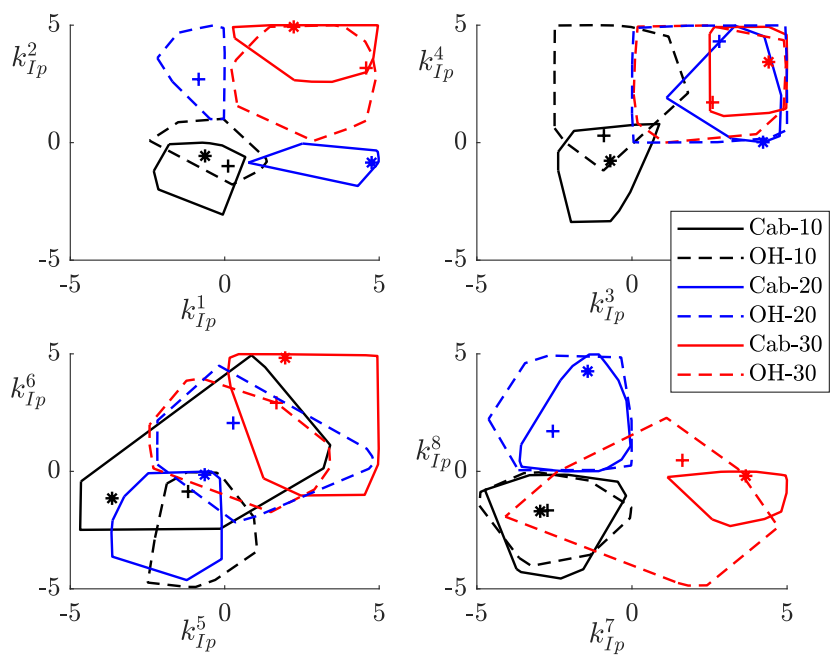

Fig. 5. Optimal values of ramp parameters $k_{I p}^{i}$ with their associated DPS for different analyzed scenarios in case of fault F2 at bus 1044

differ significantly compared to the rest of the search space, i.e. moving the parameter settings inside the DPS would not create substantial changes in the objective function compared to moving outside of the DPS. This also means that the sensitivity of the objective function value to the parameters settings is directly related to the size of the DPS: the smaller the DPS, the larger the sensitivity of the objective to the parameters, and vice versa. Applying this to the results presented in Fig. 4 for fault F1, it can be concluded that the parameters $k_{I p}^{1}, \ldots, k_{I p}^{4}$ of the IBGs located on the feeders connected to the observed bus 1041 have higher impact on the speed of voltage recovery at bus 1041 since the projection of DPS on their parameter space is smaller than the projection on the parameter spaces of $k_{I p}^{5}, \ldots, k_{I p}^{8}$ relative to the IBGs located on the feeders connected to bus 1043 . This can partly be explained by higher installed capacity of the IBG groups connected at bus 1041 feeders. Similar trend can be observed for DPS in Fig. 5 obtained for the case of fault F2 at bus 1044 .

Comparing the locations of the DPS in Fig. 4 and Fig. 5 for the different analyzed scenarios, it can be observed that optimal $k_{I p}^{i}$ values depend highly on the ratio of dynamic load component in the system. With the higher percentage of dynamic load component, the optimal $k_{I p}^{i}$ values indicate that ramping up the active current during the fault will result in faster voltage recovery. This is especially pronounced for the parameters $k_{I p}^{1}, \ldots, k_{I p}^{4}$ which also, based on the previous conclusions, tend to have more impact on the speed of voltage recovery than parameters $k_{I p}^{5}, \ldots, k_{I p}^{8}$. The similar tendency can be observed for optimal settings of $k_{I p}^{5}, \ldots, k_{I p}^{8}$ in the cables case scenarios. In the overhead case scenarios, optimal settings of $k_{I p}^{5}, \ldots, k_{I p}^{8}$ show higher active current ramping for $10 \%$ than for the $20 \%$ dynamic load component ratio. This can indicate that in the case of lower dynamic load component ratios, in the areas that are not highly loaded, the reactive current injection tends to be more important than active current injection during the fault. However, if the dynamic load component increases further, the active current injection becomes more important 

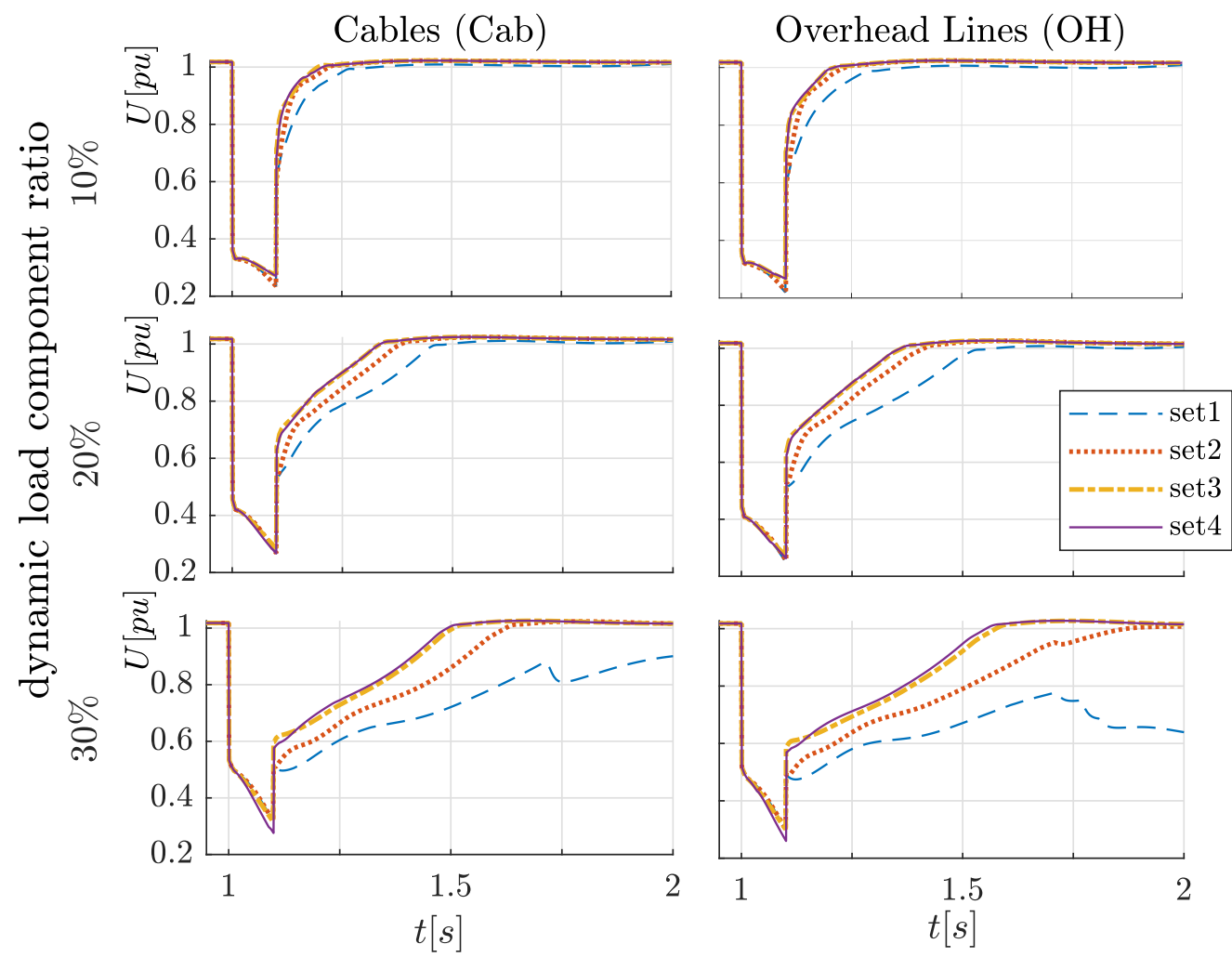

Fig. 6. Voltage response at bus 1041 in case of fault F1 for different $k_{I p}^{i}$ parameter settings (set1 - set4)

during the fault.

If the DPS of the cables scenarios are compared with the overhead lines ones, it can be concluded that the impact of the ratio of the dynamic load component to the optimal $k_{I p}^{i}$ settings is noticeably higher than the impact of the type of feeder lines installed in the area. This can be observed in Fig. 4 and Fig. 5 from the fact that the projections of DPS for the same ratio of the dynamic load component but for the different physical realizations of the feeders (cables or overhead lines) are much closer to each other.

The voltage recovery responses at bus 1041 for the studied scenarios are shown in Fig. 6 for the case of fault F1 and in Fig. 7 for the case of fault F2. For the sake of comparison, the voltage responses are given having four different values of ramp coefficients $k_{I p}^{i}$ :

set1: $k_{I p}^{i}=-10$ for all IBGs $i$ with slow recovery of the current after the clearing of the fault $\left(t_{2}=1 s\right)$;

set2: $k_{I p}^{i}=-10$ for all IBGs $i$ with fast recovery of the current after the clearing of the fault $\left(t_{2}=0 \mathrm{~s}\right)$;

set3: $k_{I p}^{i}=0$ for all IBGs $i$ (keeping active current injections constant during the fault) with fast recovery of the current after the clearing of the fault $\left(t_{2}=0 \mathrm{~s}\right)$;

set4: $k_{I p}^{i}$ for all IBGs $i$ tuned to optimal values as given in Fig. 4 with fast recovery of the current after the clearing of the fault $\left(t_{2}=0 s\right)$.

Ramp coefficient settings set1 and set 2 correspond to full cutoff of IBG active current in favor of increasing reactive current during the fault commonly suggested by grid codes.
As can be seen from Fig. 6 and Fig. 7, the dynamic load component ratio has more significant impact on the voltage recovery than the type of feeders installed. Further on, it can be concluded that the importance of appropriate control of IBGs active fault-currents rises with dynamic load component ratio as the voltage responses tend to have higher sensitivities to IBGs active fault-current control. This is the most pronounced in the case of overhead lines scenario with $30 \%$ of dynamic load component. In this case, voltage collapse at bus 1041 happens if the active current is cut-off during the fault and recovered after one second (set1).

Comparing the responses for different ramp coefficients $k_{I p}^{i}$ values, it can be concluded that the optimal voltage recovery response does not differ very much from the response having all IBGs on-fault active currents constant. This observation leads to the conclusion that the simple setting of keeping IBGs active currents constant, especially in the heavy loaded areas as bus 1041, would result in good enough voltage recovery of the area. The additional ramping up of active current during the fault is resulting in faster, but not much faster voltage recovery. On the other hand, cutting-off of active current in favor of reactive current injection, as suggested by some grid codes, can lead to delayed voltage recovery or even voltage instability.

\section{Robustness Analysis}

The previously presented results have been obtained assuming perfect information on the system parameters. In reality, this information may be very hard to obtain. Therefore, it is of 

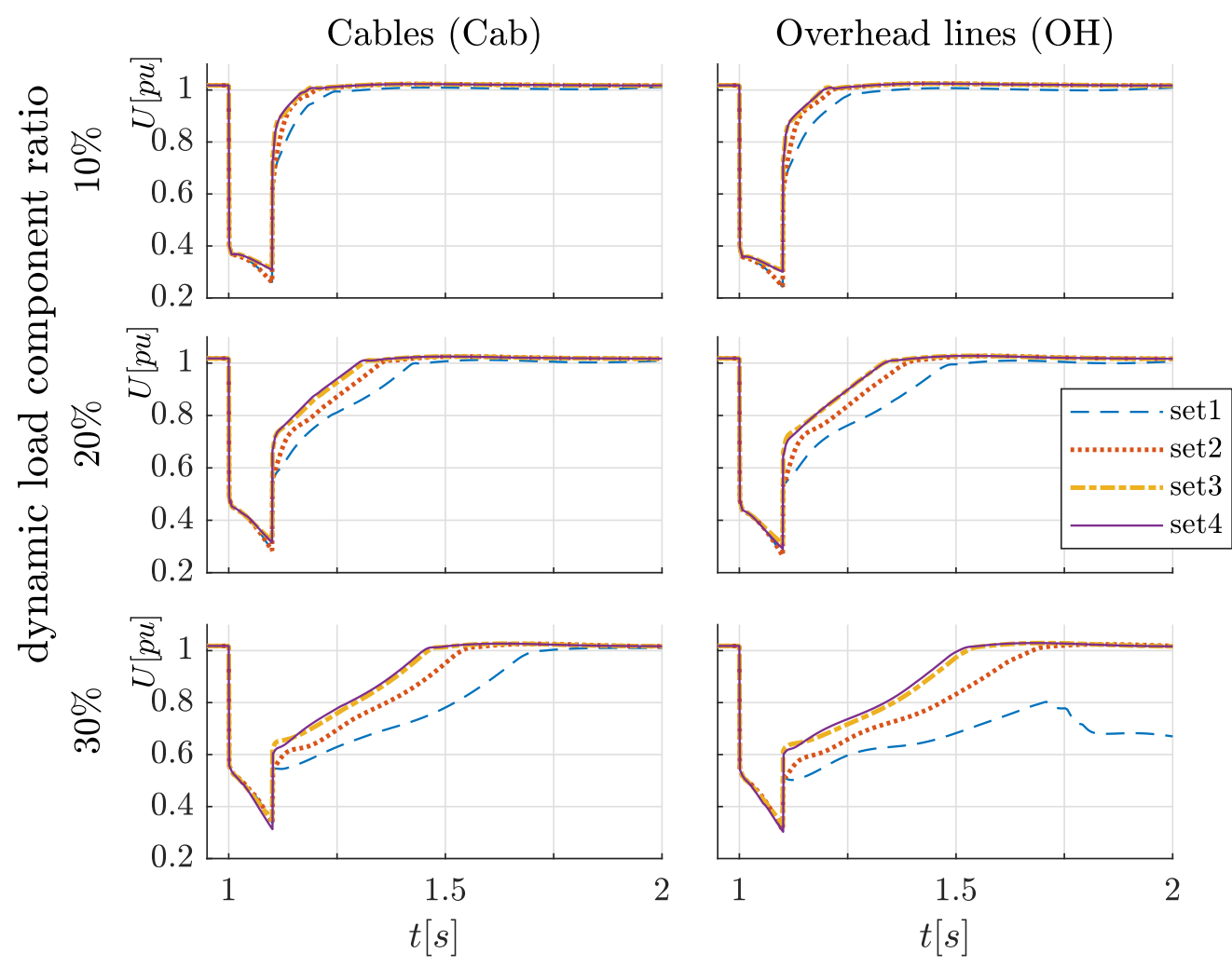

Fig. 7. Voltage response at bus 1041 in case of fault F2 for different $k_{I p}^{i}$ parameter settings (set1 - set4)

interest to analyze how robust the optimal settings of proposed $\mathrm{Ip} / \mathrm{Iq}$ controllers are to the uncertainty of certain parameters of the model. As the previous analyzes showed significant sensitivity of voltage response to the ratio of dynamic load component, the robustness analysis focuses on the uncertainty of load parameters in the system.

In order to analyze this uncertainty, the Monte Carlo method is used for sampling different possible combinations of load parameters values. The parameters are uniformly varied in two different ranges assuming no correlation between the various parameters of the given load:

1: ranges according to [19] as given in Table VI;

2: $+/-20 \%$ range from initially assumed parameters values given in Table I.

Furthermore, two different correlations between the loads connected at different buses of the system are assumed:

a: no correlation;

b: all the loads in the system have the same parameters.

The $k_{I p}$ parameters of the IBG Ip/Iq controllers are tuned

TABLE VI

RANGES OF PARAMETER VALUES OF THE INDUCTION MACHINE MODELS TO BE VARIED TAKEN FROM [19]

\begin{tabular}{|c|c|c|c|c|c|c|c|c|c|}
\hline \multirow{2}{*}{$\begin{array}{l}\text { parameters } \\
\text { range }\end{array}$} & \multicolumn{7}{|c|}{ dynamic loads (induction machines) } & \multicolumn{2}{|c|}{ static load } \\
\hline & Rs & Lls & $\mathrm{Lm}$ & $\mathrm{RR}$ & Llr & $\mathrm{H}$ & LF & $\alpha$ & $\beta$ \\
\hline lower value & 0.013 & 0.06 & 1.8 & 0.008 & 0.06 & 0.4 & 0.4 & 1 & 1.5 \\
\hline higher value & 0.130 & 0.15 & 4.0 & 0.130 & 0.20 & 2.0 & 0.9 & 2 & 3 \\
\hline
\end{tabular}

to provide fastest voltage recovery for the originally assumed load parameters values given in subsection II-A. As a stopping criteria for the Monte Carlo analysis, a check is made of how much the distribution of samples of the objective function from (1) is changing. If the change of this distribution is below certain threshold, the simulation is stopped.

The cable case scenario with $20 \%$ of dynamic load component (Cab-20) with optimally tuned IBGs Ip/Iq controllers is chosen, from which the Monte Carlo analysis is run. Fig. 8 shows voltage recovery responses at bus 1041 assuming respectively no correlation between the loads and all loads in the system with the same parameter values. The comparison is made between the initial voltage evolution, the best and the worst response samples for $\pm 20 \%$ and full range of load parameters variation as given in Table VI.

Looking at the left plot in Fig. 8, it can be seen that independent randomization of load parameters leads to improved voltage recovery as the Monte Carlo method couldn't find a sample with a worse voltage response than the initial. On the other hand, with the assumption that all loads in the system have the same parameter values, the Monte Carlo method was able to find samples with worse voltage recovery, as confirmed by the right plot in Fig. 8 .

Next, the proposed Ip/Iq controllers have been optimally re-tuned for the sample of load parameters with the worst voltage recovery. The results are compared in Fig. 9 with those of the initially optimal controller settings. It can be seen from Fig. 9 that the DPS of the two cases overlap in most dimensions except $k_{I p}^{1}$. In other words, the optimal points 

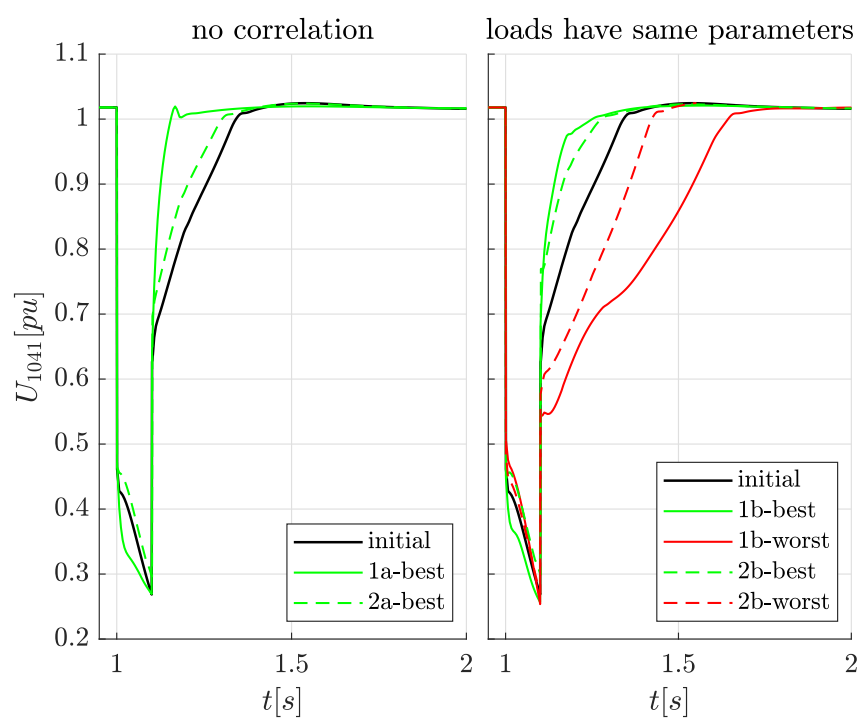

Fig. 8. Voltage recovery responses for different analyzed scenarios of load parameters uncertainty
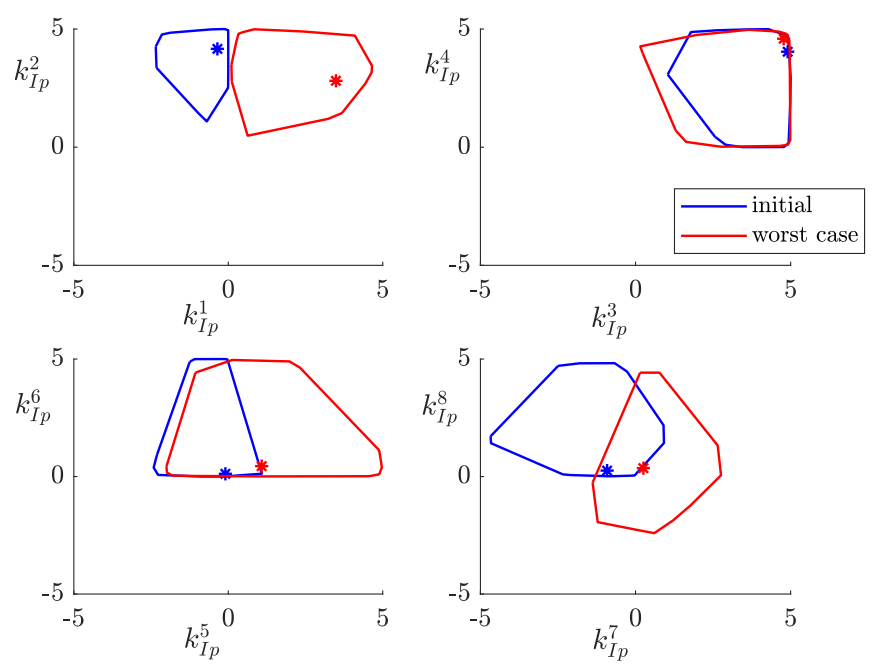

Fig. 9. Optimal values of ramp parameters $k_{I p}^{i}$ with their associated DPS for the worst sample from Monte Carlo analysis

of both cases have similar value except for $k_{I p}^{1}$. From the corresponding voltage responses given in Fig. 10 it can be seen that the difference between the two settings is minor: indeed, the newly optimized parameters give only a very slight improvement of the response shown in dashed blue line. This means that the increase of the value of $k_{I p}^{1}$ in the newly optimized settings does not have a big impact on the voltage response. Therefore, the originally tuned parameters of the controller give a near optimal response also for the worst case combination of uncertain load parameters.

The uncertainty on the load parameters affects the optimal response, which makes load model parameter estimation essential in voltage recovery studies. A further proof of this is obtained by comparing the voltage recoveries in the Cab20 scenario with respectively the initially assumed and the worst sample of load parameters values found by the Monte

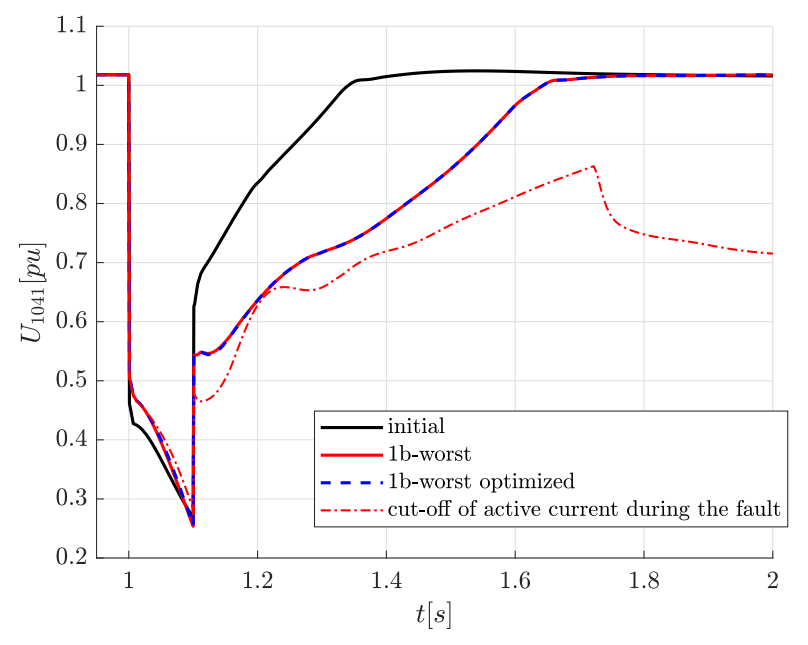

Fig. 10. Optimal voltage recovery responses for initially and worst case combination of load parameters

Carlo analysis. In the case of the optimally tuned IBG current controller, the response deteriorates as can be seen by comparing the dashed blue with the solid black line in Fig. 10. If instead of resorting to the optimally tuned IBG controller, the active fault-currents are cut off, some of the induction motors stall resulting in depressed voltage at bus 1041 and subsequently over the whole radial grid connected to it. This case is represented with dot-dashed red line in Fig. 10. For the initially assumed load parameters and the same settings of IBG current controller (identified as set1 in subsection III-C), the system is not experiencing voltage instability, as can be seen from Fig. 6 in case of fault F1 and Fig. 7 in case of fault F2.

\section{Discussion}

The results from the case studies show strong correlation between optimal settings of IBG current controllers and ratio of dynamic loads in the analyzed area. In real life applications, the optimal tuning of the proposed IBG current controllers might be challenging as it relies on the very good information about system parameters and advanced telecommunication structure in the power system. However, the results show that there exists a relatively wide region in which the controller parameters can be and still give near optimal performance. A practical recommendation drawn from the observed results is that the active current levels of the IBGs located in medium or heavy load areas should not be decreased during the fault if the nearby loads dynamic component ratios are higher than some threshold e.g. $\gamma=20 \%$. This conclusion could complement some of the existing grid codes which give more universal guidelines on tuning of IBGs fault-current behavior without considering loads composition.

The load model used in this paper consists of a static polynomial component and a dynamic load component given as a combination of two types of induction machines. Compared to the commonly used WECC composite load model [29], this model includes only two types of induction motors and 
does not explicitly model electronic loads. Having four instead of two motors in the model should not affect the obtained results in a great manner. Such observation can be made from Subsection III-D which shows that the optimal settings of the IBG current controllers are more sensitive to the total ratio of dynamic load component than particular parameter values of individual dynamic load components. In WECC composite load model, electronic loads are represented as constant power loads [29]. As such, they are not explicitly included in the load model used in this paper but they can be considered as a part of static exponential load component. From this point of view, not including explicitly constant power electronic loads should not have a big impact on the obtained results of this paper. However, electronic loads may behave differently in practice depending on the internal process control settings [30]. Further studies should take into account this fact as well as other load components with their specific behaviors and dynamics.

The analysis in this paper assumed IBGs as standalone units or aggregated groups modeled as single IBGs. In the case of the latter, the question arises on how should the individual IBGs in these groups be controlled to achieve a desirable response of the aggregated group as calculated in this paper. This can be done by formulating an optimization problem whose objective function reflects the deviation of the aggregated response of individual IBGs from the response of a single IBG equivalent, as used in this paper. Decision variables of the problem would then be individual IBGs controller parameters. This is somewhat an inverse process of making ADN equivalents as described in [14], [20]. If an aggregated group represents a microgrid, an individual contribution of each hosted IBG can be found by using the procedures described in [7], [8]. In this case, the calculated aggregated IBG responses in this paper can be used as reference active and reactive power exchanges between a microgrid and the overlying system.

\section{CONClusion}

The analyses presented in this paper show that IBGs active fault-currents have significant impact on the speed of voltage recovery. This impact is especially pronounced in the cases where the dynamic load component ratio is larger than $20 \%$. The correlation between the ramping settings of the IBGs active fault-currents resulting in the fastest voltage recovery and the dynamic load component ratio has been demonstrated. It has been also shown that decreasing the IBGs active currents during the fault can have a negative impact on voltage recovery, and even sometimes result in voltage instability. The future work should include experimental tests extending the scope and validating the approach of this paper.

The results of this paper show that the IBGs fault-current behavior should be designed having in mind the size and composition of the loads located in the same area as IBGs. With respect to this, an update of current European grid codes is suggested, in so far as they recommend some IBG control without consideration of the load dynamics.

\section{REFERENCES}

[1] Technical Connection Rules for Medium-Voltage (VDE-AR-N 4110), 2017.

[2] Technical Connection Rules for High-Voltage (VDE-AR-N 4120), 2018.

[3] The Grid Code, National Grid, 30 September 2016. [Online]. Available: http://www2.nationalgrid.com/uk/industry-information/ electricity-codes/grid-code/the-grid-code/

[4] Fingrid, Frid Code Specifications for Power Generating Facilities VJV2018, 2018.

[5] EirGrid Grid Code, 2020.

[6] Energinet, Requirements for Grid Connection of New Generation Facilities - Appendix 1 - Requirements, 2019.

[7] H. R. Baghaee, M. Mirsalim, G. B. Gharehpetian, and H. A. Talebi, "A new current limiting strategy and fault model to improve fault ridethorugh capability of inverter interfaced ders in autonomous microgrids," Sustainable Energy Technologies and Assessments, vol. 24, pp. 71-81, Dec. 2017.

[8] — , "Oc/ol protection of droop-controlled and directly voltagecontrolled microgrids using tmf/ann-based fault detection and discrimination," IEEE Journal of Emerging and Selected Topics in Power Electronics, vol. 9, no. 3, pp. 3254 - 3265, Dec. 2019.

[9] B. Weise, "Impact of k-factor and active current reduction during fault-ride-through of generating units connected via voltage-sourced converters on power system stability," IET Renewable Power Generation, 2014.

[10] G. Lammert, D. Premm, L. D. P. Ospina, J. C. Boemer, M. Braun, and T. Van Cutsem, "Control of photovoltaic systems for enhanced short-term voltage stability and recovery," IEEE Trans. Energy Convers., vol. 34, no. 1, 2019.

[11] R. K. Varma and S. Mohan, "Mitigation of fault induced delayed voltage recovery (fidvr) by pv-statcom," IEEE Trans. Power Syst., vol. 35, no. 6, 2020.

[12] K. Kawabe, Y. Ota, A. Yokoyama, and K. Tanaka, "Novel dynamic voltage support capability of photovoltaic systems for improvement of short-term voltage stability in power systems," IEEE Trans. Power Syst., vol. 32, no. 3, 2017.

[13] B. Qi, K. N. Hasan, and J. V. Milanović, "Identification of critical parameters affecting voltage and angular staiblity considering loadrenewable generation correlations," IEEE Trans. Power Syst., 2019.

[14] G. Chaspierre, G. Denis, P. Panciatici, and T. Van Cutsem, "An active distribution network equivalent derived from large-disturbance simulations with uncertainty," IEEE Trans. Smart Grid, vol. 11, no. 6, 2020.

[15] S. Stanković and L. Söder, "Optimal power flow based on genetic algorithms and clustering techniques," in Power Syst. Comp. Conf. (PSCC), 2018.

[16] S. Dasgupta, M. Paramasivam, U. Vaidya, and V. Ajjarapu, "Entropybased metric for characterization of delayed voltage recovery," IEEE Trans. Power Syst., vol. 30, no. 5, 2015.

[17] T. Van Cutsem and C. Vournas, Voltage Stability of Electric Power Systems. Springer, 1998.

[18] M. G. L. Prabha Kundur, Neal J. Balu, Power system stability and control. McGraw-Hill, 1994.

[19] C. W. Taylor, Power System Voltage Stability. McGraw-Hill, 1994.

[20] G. Chaspierre, P. Panciatici, and T. Van Cutsem, "Dynamic equivalent of a distribution grid hosting dispersed photovoltaic units," in 10th Bulk Power Syst. Dyn. Control Symp. (IREP), 2017.

[21] E. D. Tuglie, M. Dicorato, M. L. Scala, and P. Scarpellini, "A static optimization approach to assess dynamic available transfer capability," IEEE Trans. Power Syst., vol. 15, pp. 1069-1076, 2000.

[22] A. Tiwari and V. Ajjarapu, "Optimal allocation of dynamic var support using mixed integer dynamic optimization," IEEE Trans. Power Syst., 2011.

[23] M. Paramasivam, A. Salloum, V. Ajjarapu, V. Vittal, N. B. Bhatt, and S. Liu, "Dynamic optimization based reactive power planning to mitigate slow voltage recovery and short term voltage instability," IEEE Trans. Power Syst., vol. 28, no. 4, 2013.

[24] P. Aristidou, D. Fabozzi, and T. Van Cutsem, "Dynamic simulation of large-scale power systems using a parallel schur-complement-based decomposition method," IEEE Trans. Parallel Distrib. Syst., 2014.

[25] "Test systems for voltage stability analysis and security assessment," IEEE Power and Energy Society, Tech. Rep., 2015.

[26] M. Melanie, An Introduction to Genetic Algorithms. MIT Press, 1996.

[27] S. N. Sivanandam and S. N. Deepa, Introduction to Genetic Algorithms. Springer, 2008. 
[28] M. Ester, H.-P. Kriegel, J. Sander, and X. Xu, "A density-based algorithm for discovering clusters in large spatial databases with noise," in Second Int. Conf. on Knowledge Discovery and Data Mining, 1996.

[29] “Wecc mvwg load model report ver. 1.0," WECC, Tech. Rep., Jun. 2012.

[30] M. Rylander, W. M. Grady, A. Arapostathis, and E. J. Powers, "Power electronic transient load model for use in stability studies of electric power grids," IEEE Trans. Power Syst., vol. 25, no. 2, May 2010. 